\title{
LOS GUSANOS DE SEDA (BOMBYX MORI) COMO RECURSO IDÓNEO PARA EL APRENDIZAJE POR INDAGACIÓN EN EL GRADO DE EDUCACIÓN INFANTIL \\ Recepción: 19/03/2019 | Revisión: 03/04/2019 | Aceptación: 10/08/2019
}

\section{Jerónimo TORRES-PORRAS}

Universidad de Córdoba

jeronimo.torres@uco.es

Resumen: Es necesario fomentar la ciencia en la sociedad actual y para ello se puede y debe comenzar en las primeras etapas educativas. Sin embargo, para que docentes de Educación Infantil sean capaces de enseñar Ciencia haciendo ciencia, deben haberla experimentado en su etapa formativa en la universidad. En este artículo se analiza la idoneidad de utilizar los gusanos de seda como recurso para fomentar la indagación en el grado de Educación Infantil. Se muestra una experiencia en la que el alumnado ha tenido que realizar un estudio científico del ciclo de los gusanos de seda y proponer su aplicación a las aulas de Educación Infantil. Los resultados son muy positivos: el alumnado realizó distintas propuestas de investigación sobre la especie y argumentó que la indagación ha sido muy interesante, que ha aumentado su interés por la ciencia y que utilizaría esta metodología en su futura labor docente.

Palabras clave: indagación; seres vivos; método científico; alfabetización científica; enseñanza de las ciencias.

\section{SILKWORMS (BOMBYX MORI) AS IDEAL RESOURCE FOR INOUIRY-BASED LEARNING INTHE EARLY CHILDHOOD EDUCATION DEGREE}

\begin{abstract}
It is necessary to promote science in our current society and so this promotion both can and should begin in the earlier stages of education. However, for teachers of Early Childhood Education to be able to teach Science doing science, they need to have done science in their formative stage, that is, at university. This article analyzes the suitability of using silkworms as a resource to promote inquiry in the degree in Early Childhood Education. It shows an experience in which the students had to study the silkworm cycle and propose its application in the Early Childhood classrooms. The results are very positive: the students made different research proposals about the species, and they exposed that it had been very interesting. Besides, they said their interest in science had increased and that they would use this methodology in their future teaching work.
\end{abstract}

Keywords: inquiry; living beings; scientific method; scientific literacy; science teaching.

\section{ELS CUCS DE SEDA (BOMBYX MORI) COM A RECURS IDONI PER A L'APRENENTATGE PER INDAGACIÓ AL GRAU D'EDUCACIÓ INFANTIL}

Resum: Cal fomentar la ciència a la societat actual. Amb aquest objectiu, es pot $i$ s'ha de començar a fer durant les primeres etapes educatives. No obstant això, perquè el docent d'Educació Infantil sigui capaç d'ensenyar Ciència fent ciència, ha d'haver-la experimentat durant la seva etapa formativa a la universitat. En aquest articles s'analitza la idoneïtat d'utilitzar els cucs de seda com a recurs per fomentar la indagació al grau d'Educació Infantil. Es mostra una experiència en la que lalumnat ha hagut de realitzar un estudi cientific del cicle dels cucs de seda i proposar-ne l'aplicació a les aules d'Educació Infantil. Els resultats són molt positius: l'alumnat va realitzar diferents propostes d'investigació sobre l'espècie i va argumentar que la indagació havia estat molt interessant, que havia augmentat el seu interès per la ciència i que utilitzaria aquesta metodologia en la seva futura tasca docent.

Paraules clau: indagació; éssers vius; mètode científic; alfabetització científica; ensenyament de les ciències. 


\section{Introducción}

La necesidad de conocer y comprender la ciencia en la sociedad actual es evidente y la alfabetización científica de la ciudadanía, así como fomentar el alcance de la competencia científica es una prioridad en la educación (Acevedo, 2004). Distintos autores e informes advierten de la necesidad de trabajar la ciencia desde las etapas tempranas de la educación, comenzando por la educación infantil (Cañal, 2006; COSCE, 2011; Spektor-Levy, Kesner, y Mevarech, 2013), lo que queda reflejado en los diferentes currículos escolares.

Los gusanos de seda (Bombyx mori) se han propuesto por algunos autores como una herramienta para trabajar los seres vivos y las ciencias experimentales en educación, especialmente en Educación Infantil, donde se sugieren actividades (Bentley y Auldridge, 2017; Torres-Porras, 2017), estando contemplado en el currículo de esta etapa educativa la necesidad de trabajar el contacto con otros animales (Orden ECI/3960/2007).

Se trata de insectos pertenecientes al grupo de los lepidópteros cuyos adultos son mariposas que se reproducen poniendo huevos de donde surgen las fases larvarias llamadas orugas (Martínez-Ramírez, 2002; Rodríguez et al., 2012), o mal nombrados gusanos, ya que los gusanos son otros grupos de invertebrados. Son adecuados ya que son fáciles de mantener, es común su cría en las escuelas o por parte de las niñas y niños en sus casas y, además de permitir el acercamiento a los animales en general y a los insectos en particular, podrían facilitar una metodología activa de aprendizaje de la ciencia.

El principal objetivo de este trabajo es analizar si los gusanos de seda son un recurso idóneo para que el alumnado del grado de Educación Infantil se acerque al método por indagación, haciendo ciencia, de forma que lleven a cabo una investigación real sobre la biología de una especie fácil de mantener y diseñen actividades para realizar con el alumnado de Educación Infantil, para que posteriormente puedan aplicar esta metodología a las aulas escolares. Para ello planteamos estos tres objetivos específicos: (1) estudiar el ciclo de vida de los gusanos de seda; (2) analizar las investigaciones y las propuestas de los docentes en formación para trabajar el ciclo de vida de esta especie en Educación Infantil, y (3) estudiar las opiniones de dichos docentes en formación sobre la metodología de trabajo utilizada en esta experiencia.

Podemos decir que la gran mayoría conoce o ha criado esta especie y podría repetir la experiencia en Educación Infantil, pero el objetivo es que los estudiantes aprendan a aplicar la ciencia a la observación del ciclo de los gusanos de seda en esta etapa educativa, base fundamental para el posterior desarrollo de la competencia científica.

\section{Marco teórico}

Los niños y niñas tienen una tendencia natural a observar el medio que les rodea, por lo que hay que aprovechar esta predisposición para crear entornos apropiados de aprendizaje de las ciencias (French, 2004). Si el profesorado guía a su alumnado de forma adecuada, puede ayudarlo a construir su propio conocimiento científico (Duschl, Schweingruber y Shouse, 2006; Nayfeld, Brenneman y Gelman, 2011). No se trata, por lo tanto, de trasmitir ciencia o conocimiento a las 
niñas o niños, sino que el conocimiento sea construido y adquirido por los propios infantes. El profesorado tiene que crear un ambiente de aula adecuado, con tiempo y materiales que inciten a la investigación y fomenten la ciencia escolar. Debe realizar preguntas productivas (Martens, 1999), que sirvan para conectar lo que saben con lo que están observando. No se trata tampoco de facilitar las respuestas a sus preguntas, sino de aportar las herramientas y el ambiente necesario para que el alumnado se haga preguntas y se pueda plantear entre todos cómo buscar las respuestas a esas preguntas.

Estas experiencias positivas y tempranas sobre ciencia pueden promover actitudes positivas hacia esta y una base para comprender conceptos científicos en el fututo (Eshach y Fried, 2005). Encontramos distintas propuestas que muestran metodologías activas y participativas para trabajar las Ciencias Experimentales en Educación Infantil (Briones Fernández y Torres-Porras, 2016; Fernández-Oliveras, Molina-Correa, y Oliveras, 2016; Gómez-Montilla y Ruíz Gallardo, 2016; Cruz-Guzmán, García-Carmona, y Criado, 2017).

Sin embargo, aunque en la comunidad científica exista un consenso en la forma en la que se debe trabajar la ciencia en Educación Infantil, la realidad observada es que todavía quedan problemas por solucionar, destacando la ausencia de actividades claves para aprender ciencias (Cantó Doménech, de Pro Bueno y Solbes, 2016), trabajándose las ciencias de forma no reflexiva y por consiguiente no ayudando a desarrollar el razonamiento científico. Entre las causas encontramos que distintos docentes de educación infantil conciben la ciencia como algo complejo con gran cantidad de contenidos y no como algo dinámico en donde el proceso es lo importante, lo que implica que su motivación para trabajar ciencias sea reducida o no se sientan con capacidad para abordarlas (Siry, 2013; Spektor-Levy, Kesner, y Mevarec, 2013).

Los Grados de Educación son fundamentales para acercar la ciencia a los futuros docentes, trabajando una ciencia motivadora con el objetivo de alcanzar la competencia científica del alumnado (Pedrinaci, Caamaño, Cañal y de Pro, 2013), tratando de fomentar la curiosidad y el interés, así como mostrar su aplicación práctica a las aulas escolares (Aragón Núñez et al., 2016). Hay que trabajar en los grados como se ha comentado anteriormente, haciendo ciencia. Que el profesorado universitario actúe como guía del aprendizaje del alumnado de los grados, fomentando la observación, el planteamiento de interrogantes, cómo resolverlos y la obtención de conclusiones. Se debe trabajar la ciencia de forma práctica implicando al alumnado en ella; de hecho, existen distintas experiencias en los grados de educación que muestran un acercamiento activo a la ciencia a través de la indagación en el aula (Cortés Gracia y De la Gándara Gómez, 2007; Gil Quilez et al., 2008; Torres-Porras y Villamandos de la Torre, 2015; Gil, Martínez, y Cordero, 2017).

Se considera que la indagación tiene un gran potencial en el aprendizaje de las ciencias (Cañal de León, 1999; Romero-Ariza, 2017) y que se debe fomentar su uso en las distintas etapas educativas. Sin embargo, los trabajos prácticos suelen tener un bajo nivel de indagación, ya que no es el alumnado el que plantea las preguntas, sino el profesorado (Tamir y García, 1992). Distintos autores coinciden en la importancia del proceso de indagación y en que lo fundamental para comenzar es el planteamiento de preguntas investigables (Martin-Hansen, 2002; Lederman et al., 2014), para posteriormente tomar datos que permitan responderlas. Se deben diseñar entonces 
proyectos que posibiliten al alumnado a realizar e identificar cuestiones que puedan derivar en investigaciones (Sanmartí y Márquez, 2012).

A pesar de que la indagación en el aula es uno de los mejores métodos para trabajar las ciencias, mejorando la motivación y actitud hacia la práctica científica del alumnado (Bevins y Price, 2016), casi no está instaurada en las aulas (Osborne y Dillon 2008; COSCE, 2011). Por lo tanto, será necesario trabajar esta metodología con los maestros en formación de forma que posteriormente tengan las herramientas necesarias para poder desarrollarla en sus aulas.

\section{Metodología y descripción de la experiencia}

Este trabajo se ha realizado en la asignatura de Didáctica de las Ciencias Naturales y Sociales en Educación Infantil del tercer curso del Grado de Educación Infantil de la Universidad de Córdoba con un grupo clase de 70 estudiantes ( 4 alumnos y 66 alumnas) durante el segundo cuatrimestre, en el que imparte docencia el autor.

Podemos distinguir tres procesos diferenciados en este trabajo, por una parte, (1) la investigación realizada sobre el ciclo de vida de la especie; por otra parte, (2) la experiencia de indagación y transposición didáctica realizada por el alumnado y, en tercer lugar, (3) una evaluación sobre dicha experiencia, mediante un cuestionario.

\subsection{Estudio del ciclo de vida}

En la investigación sobre la especie, el profesorado, a la vez que su alumnado, mantuvo unos cuantos ejemplares de gusanos de seda y realizó un seguimiento de su ciclo, lo que le permitía conocer el proceso que iba a observar el estudiantado y determinar qué tipos de datos y conclusiones se pueden obtener sobre la especie.

Los huevos del año anterior fueron mantenidos en el cartón al que estaban adheridos y situados en una caja de cartón suministrando hojas de morera (Morus alba) diariamente y eliminando las del día anterior. Cada día se anotaba la fecha y el número de orugas, en su momento el número de capullos, así como el número de mariposas.

Como parte de este estudio, se apartaron en otra caja un grupo de 17 orugas nacidas el mismo día (13 de abril), para realizar un seguimiento detallado, que permite establecer el tiempo dedicado a cada fase desde el nacimiento, así como otras variables (crecimiento, mortalidad, longevidad, número de parejas). Se medían tres orugas cada 2-6 días. Cuando hacían el capullo, se anotaba un número en la misma caja junto al capullo y se registraba la fecha y cuando emergía la mariposa, se anotaba la fecha y se le ponía un punto de color en el ala con un rotulador, lo que permitía identificarla. Se registraban los diferentes apareamientos, anotando qué individuos se apareaban. Cuando morían, se registraba la fecha de muerte de cada ejemplar. 


\subsection{Experiencia de indagación y transposición didáctica realizada por el alumnado}

Se ha aplicado una metodología constructivista para fomentar un aprendizaje significativo en el que el profesor ha actuado como guía del proceso a seguir y ha sido el alumnado el protagonista, estando implicado activamente en la construcción de su propio aprendizaje.

En una primera sesión teórica se explicó el método científico y el proceso de elaboración y publicación de un artículo científico, para reforzar la confianza en la ciencia. El método con fases como observación y definición del problema a investigar, búsqueda de información, formulación de hipótesis, planificación de la investigación, toma de datos, análisis y obtención de conclusiones. Respecto al proceso de elaboración de un artículo, siguiendo una metodología científica y con apartados en biología como introducción, material y métodos, resultados y discusión, trasladando al estudiantado las fases posteriores de relación con las revistas, así como el proceso de revisión por pares.

Seguidamente se les propuso, como actividad de aula, que razonaran sobre la posibilidad de realizar investigaciones científicas sobre el ciclo de los gusanos de seda en las aulas de infantil, como si se tratara de una especie recién descubierta. ¿Qué tipo de preguntas podrían hacer?; ¿qué investigaciones se podrían realizar?, así entre el alumnado y el profesorado se propusieron distintas cuestiones y toma de datos para resolverlas (determinar la supervivencia, el tiempo que están en cada fase, el tamaño que alcanzan los gusanos), pero sin conocer todavía que posteriormente debían realizar un trabajo sobre esta especie, de forma que esta discusión sirviera de base para el trabajo de investigación.

Posteriormente, otro día se les indicó que tenían que realizar un trabajo escrito por grupos (4-5 estudiantes) que constaba de dos partes. Una primera parte consistía en realizar un estudio científico de los gusanos de seda, para lo que se le proporcionó a cada grupo un cartón con huevos de esta especie, pero no se les suministraba ningún protocolo, sino que tenían que aplicar los conocimientos ya tratados en clase anteriormente mencionados. Es decir, entre los distintos niveles de la enseñanza por indagación, este caso sería una indagación abierta, el nivel más complejo, en el que el alumnado plantea sus propios interrogantes y diseña sus investigaciones (Windschitl, 2003), aunque se recordaba que al comienzo de las siguientes sesiones podrían ir planteando dudas de sus diseños.

La segunda parte del trabajo era su transposición didáctica a las aulas de Infantil, es decir, debían explicar cómo trabajar el ciclo de esta especie en Educación Infantil, aplicando metodología científica.

Posteriormente a lo largo del cuatrimestre se abordaba este tema al comienzo de las clases, viendo cómo iba el proceso y respondiendo a dudas sobre la metodología a seguir. Se les facilitó a través de la plataforma virtual Moodle el acceso a un artículo sobre la biología de un lepidóptero (García-Barros, 1992), así como un mapa con localizaciones de moreras.

Se realizó una toma de datos sobre los 18 trabajos presentados por el alumnado. En la parte de investigación se determinó el porcentaje de trabajos que planteaban interrogantes; realizaban una toma de datos planificada y sistemática; medían el desarrollo de los gusanos; mostraban 
resultados; utilizaban gráficas o tablas; y obtenían y mostraban conclusiones, todos aspectos importantes en la indagación (Minner, Levy y Century, 2010). En la parte de la aplicación didáctica a Educación Infantil, para determinar si se aplicaba la indagación en sus propuestas de actividades, se determinó el porcentaje de trabajos que planteaban interrogantes para trabajar en las aulas; los que aplicaban una metodología científica para responder a dichos interrogantes; los que medían el desarrollo de los gusanos (ya que implica una toma de datos sistemática y la utilización de herramientas de medición), así como qué otros recursos o actividades adecuadas a Educación Infantil planteaban.

\subsection{Cuestionario sobre la experiencia}

Una vez terminada la experiencia, el alumnado contestó a un cuestionario en el que tenía que expresar su grado de acuerdo con una serie de afirmaciones, teniendo en cuenta que 1 significaba "Totalmente en desacuerdo" y 5 "Totalmente de acuerdo". Las afirmaciones eran las siguientes:

- Me ha resultado complicado la realización del trabajo.

- Me ha resultado interesante la realización de este trabajo.

- He aprendido el procedimiento de una investigación.

- Esta práctica ha contribuido a aumentar mi interés por la Ciencia.

- He disfrutado cuidando a los gusanos de seda.

- He aprendido sobre la biología de los gusanos de seda aspectos que desconocía.

- Creo que no es importante tener seres vivos en Educación Infantil.

- En mi futura labor docente tendré en clase gusanos de seda.

- En mi futura labor docente tendré gusanos de seda en clase y aplicaré el método científico para que mi alumnado los estudie.

Para la realización de los análisis estadísticos y las gráficas se ha utilizado el programa STATISTICA 8.0 (Statsoft Inc., Tulsa, Oklahoma, USA) y Microsoft Excel.

\section{Resultados y discusión}

Los resultados podemos estructurarlos en tres bloques, (1) resultados del estudio científico del ciclo de los gusanos de seda; (2) descripción de los trabajos realizados por el alumnado, tanto el estudio de la especie como su transposición didáctica a las aulas de Educación Infantil; (3) resultados del cuestionario realizado por el alumnado al final del proyecto.

\subsection{Resultados del estudio del ciclo de vida}

Estos resultados pretenden mostrar qué información se puede obtener al estudiar la especie. Se pueden responder a cuestiones básicas sobre la biología de los gusanos de seda, como ¿durante qué meses se producen las distintas fases de ciclo de vida?, ¿cuánto tiempo permanecen en cada 
fase?, ¿hay diferencias entre sexos?, ¿cómo es la curva de crecimiento de las orugas?; estas preguntas permiten obtener resultados mediante una planificación y toma sistemática de datos.

Los resultados respecto a la fenología de la especie se muestran en la Figura 1. Orugas desde el 3 de marzo hasta el 7 de junio que hicieron las últimas el capullo. Capullos desde el 30 de abril hasta el 10 de junio. Mariposas desde el 20 de mayo hasta el 24 de junio que murió la última. Las mariposas pusieron huevos y las primeras orugas nacidas al año siguiente lo hicieron el 14 de marzo, 11 días más tarde que el año anterior. Nacieron un total de 478 orugas de 614 huevos, lo que representa un porcentaje de eclosiones de 77,85 \%.

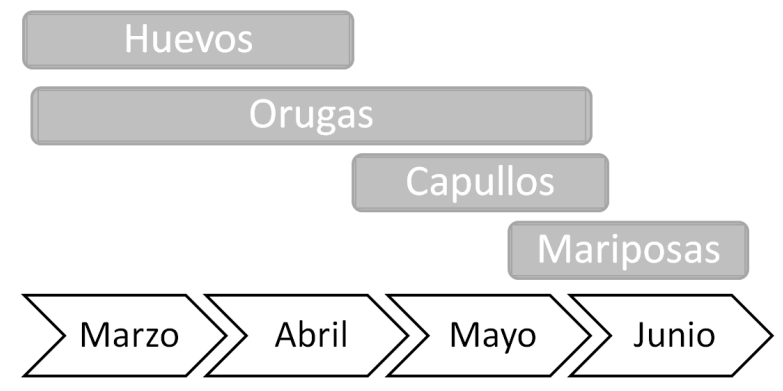

Figura 1. Fenología del ciclo de vida de los gusanos de seda. Fuente: Propia.

De las orugas nacidas el mismo día, sobre las que se realizó un seguimiento pormenorizado de su crecimiento y fases; murieron 4 orugas, lo que corresponde a un 23,53 \% de la población. La toma de datos de la longitud de las orugas permite realizar gráficas de crecimiento y observar que este es muy acusado, como se muestra en la Figura 2, siendo mayor conforme tienen más días, pasando de una longitud de $0,3 \mathrm{~cm}$ el primer día hasta $5,7 \pm 0,4 \mathrm{~cm}$ durante los últimos días.

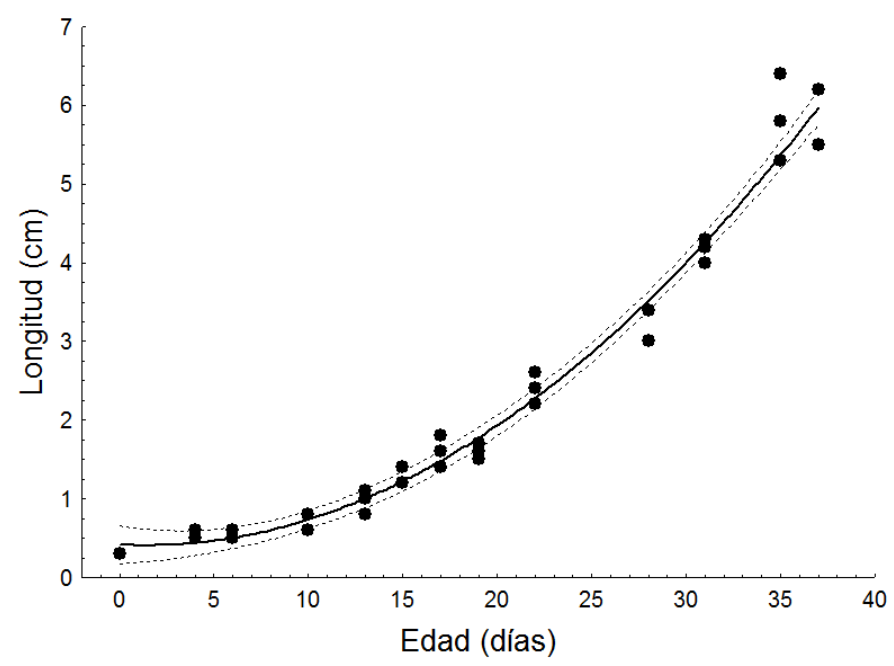

Figura 2. Regresión polinomial de la longitud de las orugas respecto a la edad en días (Intervalo de confianza al $95 \% ; y=0,41-0,0{ }^{*} x+0,004^{*} x^{2}$. Fuente: Propia. 
Han pasado una media de 38,38 \pm 1,26 días ( \pm Desviación estándar) en fase de oruga, 15 $\pm 1,68$ días en fase de capullo y 12,07 \pm 3,30 días en fase de mariposa. Las mariposas emergidas fueron 9 hembras, 3 machos y 1 indeterminado (que tuvo dificultades para salir del capullo). Es decir, más hembras que machos. Las fases han estado muy sincronizadas entre los distintos individuos, haciendo todos los capullos en un intervalo de 6 días y emergiendo en un intervalo de 7 días.

No existen diferencias significativas entre sexos en los días que han pasado en fase de capullo (Mann-Whitney U Test; $Z=1,66 ; \mathrm{p}>0,09$ ), sin embargo, sí existe en la longevidad de la fase de mariposa $(Z=2,49 ; p=0,01)$, con una media los machos de 7,0 \pm 0 días y las hembras de 13,77 \pm 1,85 días, es decir, los machos han vivido menos tiempo. Por otra parte, el número de parejas con las que se han apareado también ha variado entre sexos $(Z=-2,49 ; p=0,01)$, con una media los machos de 3,33 \pm 0,57 parejas de cópula, mientras que en las hembras la media ha sido menor, con $1,22 \pm 0,66$ parejas.

Todos estos resultados muestran lo que se puede profundizar en el estudio de la biología de una especie fácil de mantener en cautividad, tal como se podría hacer con una especie recién descubierta. También sirven para orientar a docentes que quieran implementar este proyecto en las fechas en las que se puede realizar y en la metodología y toma de datos que se puede llevar a cabo. Es decir, estos resultados sirven de guía a profesorado del grado de Educación Infantil, o también de Primaria, que quieran plantear un estudio de esta especie fomentando la indagación.

\subsection{Resultados de los trabajos realizados por el futuro profesorado}

El alumnado tenía que realizar en grupos un trabajo escrito con dos bloques, el primero un estudio científico sobre los gusanos de seda que estaban manteniendo y el segundo la transposición didáctica a las aulas de infantil del seguimiento del ciclo de vida de esta especie, desde el enfoque científico.

En el primer contacto en el aula, al alumnado le surgieron diversas preguntas (Tabla 1), y el profesorado recalcó que podrían ser preguntas para incluir en sus trabajos y que se podrían responder mediante un estudio de la especie o búsqueda bibliográfica en algunos casos, ya que no todas las preguntas son investigables con los medios disponibles.
¿Los huevos crecen?
¿Los gusanos luego se mueren?
¿Hay machos y hembras?
¿Qué comen?
¿De dónde han salido estos huevos?
¿Cómo se han pegado al cartón?
¿Cuántos huevos ponen?
¿Necesitan agua?
¿Cuándo nacen?
¿Qué cuidados necesitan los huevos y los
¿Duermen?
gusanos?
¿Son sociales, forman grupos?

Tabla 1. Preguntas formuladas por el alumnado en el aula al conocer la finalidad del trabajo.

El profesorado no respondió a esas preguntas y dejó que fuera el alumnado el que durante el desarrollo del proyecto se planteara estas y otras posibles cuestiones para responderlas. 
Respecto al trabajo de investigación, todos los trabajos (18/18) han planteado interrogantes (Tabla 2), que trataban de responder mediante la observación o documentación, algo crucial para comenzar una investigación. Además, la mayoría de estos interrogantes son preguntas investigables, con la dificultad que se suele encontrar en que el alumnado plantee este tipo de preguntas frente a las de información (Ferrés-Gurt, 2017).

¿Por qué los huevos tienen diferente color?

¿Comen solo hojas de morera o pueden comer de

otras especies? PI

¿Cada cuánto tiempo comen? PI

¿Cómo sale la mariposa? PI

¿Cuánta comida necesitan? PI

¿Cuánto tardan en nacer? PI

¿Todos los gusanos hacen a la vez el capullo? PI

¿Cómo salen del capullo? PI

¿Puede haber al mismo tiempo gusanos y

mariposas? PI

¿De qué color son las mariposas? PI

¿Hay gusanos machos y hembras?

\author{
¿Por qué hacen el capullo? \\ ¿Por qué unos capullos son blancos y otros amarillos? \\ ¿Los gusanos duermen? \\ ¿Tienen sangre? \\ ¿Por qué mudan la piel? \\ ¿Cuántas patas tienen? PI \\ ¿Cuántos huevos pone una mariposa? PI \\ ¿Cuánto tiempo viven? PI \\ ¿Son todos los gusanos del mismo color? PI \\ ¿Cuántas fases tiene el ciclo? PI \\ ¿Cuál es la tasa de supervivencia? PI \\ ¿Cuánto miden al nacer? PI \\ ¿Cuánto tiempo están en el capullo? PI \\ ¿Qué tipo de insectos son?
}

Tabla 2. Preguntas planteadas por el alumnado en sus trabajos (PI, preguntes investigables).

Once de dieciocho propusieron y realizaron una toma sistemática y planificada de datos (Tabla 3), mientras que el resto (7/18) exponía una descripción de lo observado a modo de diario, sin una planificación de toma de datos estructurada.

Algunos (3/18) planteaban diseños experimentales para responder a preguntas e hipótesis que habían propuesto, por ejemplo, respecto al tipo de alimentación: aportar hojas de distintas especies y determinar cuál es la especie seleccionada; también probar distintos microclimas, húmedo en caja de plástico y seco en una caja de cartón, observando que en el húmedo permanecen más tiempo las hojas frescas.

Una frecuencia de 7/18 de los trabajos han realizado una toma de datos del crecimiento de los gusanos, mediante mediciones a lo largo del tiempo para determinar su crecimiento y la mitad presentan tablas o gráficas para exponer los resultados, si bien todos (18/18) muestran resultados y obtienen conclusiones. Parecen resultados positivos, teniendo en cuenta que se trata de estudiantes de un grado en el que no han cursado asignaturas específicas sobre investigación, y tenían consecuentemente que aplicar los conocimientos más recientes trabajados en la propia asignatura. Es evidente, por lo tanto, que todos los grupos han planteado interrogantes, que son la base de cualquier investigación y han tomado datos para tratar de responder a las cuestiones llegando a resultados y conclusiones.

Respecto a la segunda parte de los trabajos, la aplicación didáctica a las aulas de Educación Infantil, el principal objetivo es determinar qué proporción planifican actividades de indagación. Una proporción de 13/18 plantea interrogantes para investigar en las aulas de Educación Infantil. La mitad de los trabajos (9/18) aplican una metodología científica para el seguimiento del ciclo de vida de los gusanos de seda, es decir, además de plantear interrogantes, diseñan una toma de datos 
específica para resolverlos. Esta frecuencia implica que se podría intentar mejorar mediante un seguimiento de su trabajo, para proponerles mejoras antes de finalizarlo, o aportarles algún protocolo con indicaciones de mínimos que tienen que desarrollar. El resto de los trabajos plantean la observación y cuidado de los animales. Hay una proporción de trabajos (7/18) que proponen que el alumnado realice mediciones para determinar el crecimiento de las orugas y el tamaño que alcanzan, lo que implica un seguimiento sistemático y la utilización de instrumentos de medición.

\begin{tabular}{|c|c|c|c|}
\hline \multicolumn{5}{|c|}{$\begin{array}{c}\text { Investigación especie } \\
\text { interrogantes }\end{array}$} & $\begin{array}{c}\text { Capacidad toma } \\
\text { sistemática datos }\end{array}$ & $\begin{array}{c}\text { Capacidad toma datos del } \\
\text { crecimiento }\end{array}$ & $\begin{array}{c}\text { Capacidad mostrar } \\
\text { resultados y obtener } \\
\text { conclusiones }\end{array}$ \\
\hline $18 / 18$ & $11 / 18$ & $7 / 18$ & $18 / 18$ \\
\hline \multicolumn{7}{|c|}{ Transposición didáctica a las aulas de Educación Infantil } \\
\hline interrogantes & $\begin{array}{c}\text { Capacidad diseño } \\
\text { toma de datos }\end{array}$ & $\begin{array}{c}\text { Capacidad diseño toma } \\
\text { datos del crecimiento }\end{array}$ & $\begin{array}{c}\text { Capacidad planificar otros } \\
\text { recursos }\end{array}$ \\
\hline $13 / 18$ & $9 / 18$ & $7 / 18$ & $18 / 18$ \\
\hline
\end{tabular}

Tabla 3. Capacidades mostradas por el alumnado en sus trabajos (frecuencias observadas).

Además de observar y tomar datos de los gusanos de seda, el alumnado ha descrito en sus trabajos multitud de recursos y actividades que pueden ayudar a conocer la especie (Figura 3), entre los que destaca la asamblea, y es que dialogar sobre lo que se está estudiando es fundamental para avanzar en la ciencia y para que los niños y niñas expresen sus ideas, siendo elemental por lo tanto para el aprendizaje y desarrollo de su autonomía (D’Angelo y Medina, 1997), promoviendo el hacer, pensar, hablar. Otros recursos utilizados son el cuento, las manualidades (hacer gusanos de cartón, mariposas con rollos de papel higiénico), el dibujo (sobre las distintas fases observadas), murales en donde exponer a gran tamaño el ciclo de vida, fichas sobre la especie y sus fases, imágenes de distintas fases, etc., todos ellos apropiados para esta etapa educativa y que permiten asentar y apoyar los conocimientos adquiridos mediante la observación y toma de datos. 


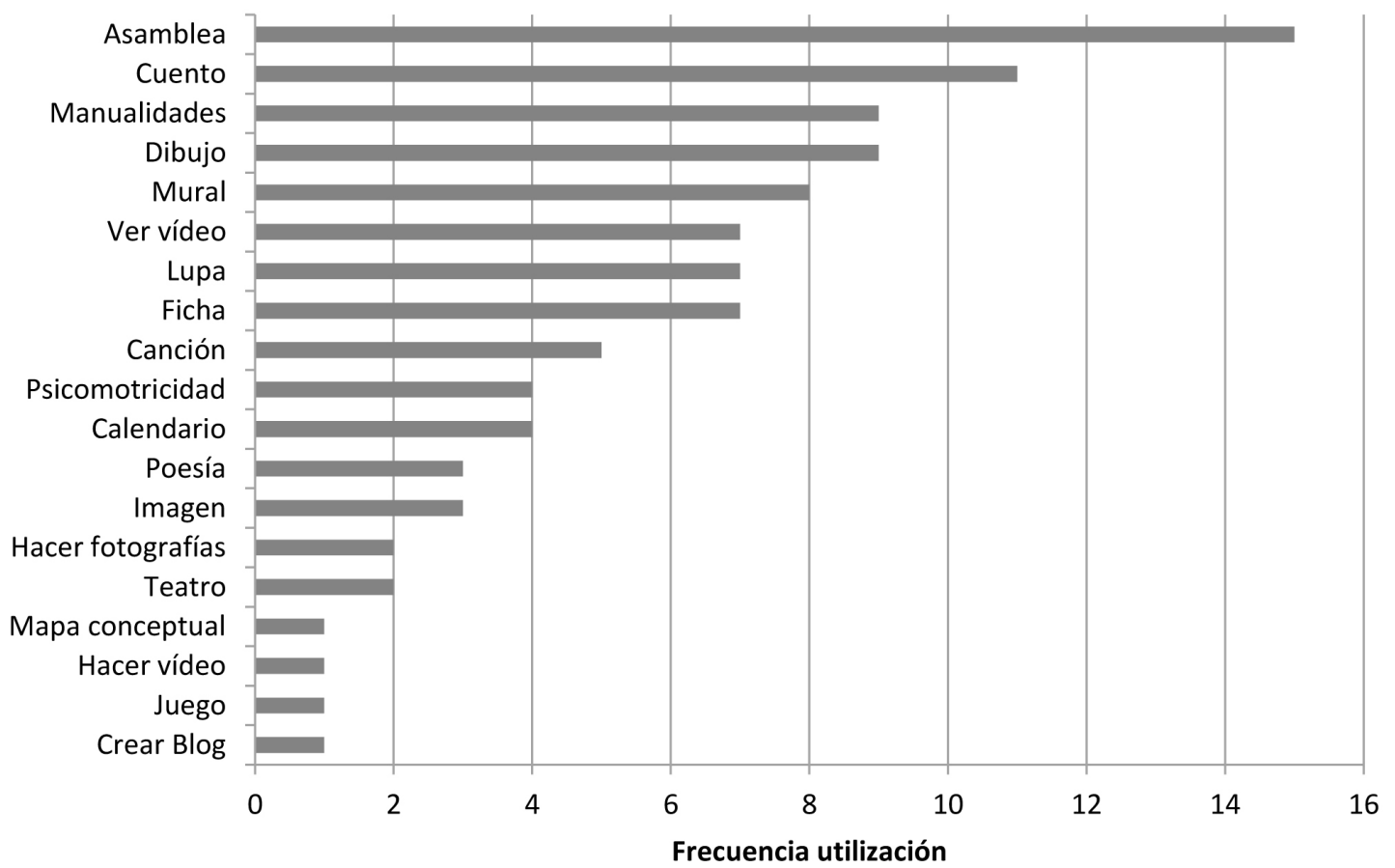

Figura 3. Recursos planteados por el alumnado en sus trabajos en las aulas de infantil (100 recursos de 18 trabajos). Fuente: Propia.

\subsection{Resultados del cuestionario final}

Al finalizar este trabajo el alumnado contestó a un cuestionario que contenía una serie de afirmaciones para poder evaluar su opinión respecto al proyecto, cuyos resultados se presentan en la figura 4. Sus respuestas respecto a la primera afirmación (complicación del trabajo), muestran una media de 2,94 \pm 1,04 (media \pm desviación estándar), es decir no están ni de acuerdo ni en desacuerdo con tal afirmación, ya que no habían realizado con anterioridad este tipo de proyectos de investigación y no se les había dado ningún guion o protocolo, lo que implicaba que les surgían muchas dudas que eran respondidas por el profesorado al comienzo de las clases o en horas de tutorías. A la mayoría les ha parecido un proyecto interesante (afirmación $2^{a}$ con una media de 4,20 $\pm 0,75$ ), consideran que han aprendido el procedimiento que se sigue en una investigación (afirmación $3^{\mathrm{a}}$ con una media de $4,22 \pm 0,81$ ) y les ha servido para aumentar su interés por la ciencia (afirmación $4^{\text {a }}$ con una media de $4,01 \pm 0,94$ ), teniendo estas tres afirmaciones medias superiores a 4 sobre 5 .

En la $5^{\text {a }}$ afirmación, si han disfrutado cuidando los gusanos de seda, la media disminuye a 3,54 $\pm 1,42$; debido fundamentalmente a que una parte del alumnado siente cierta repulsión hacia los insectos en general y hacia las larvas de estos en particular, como comentaron en clase y se ha comprobado en otros estudios (Wagler y Wagler, 2012), aunque también podría haber más razones de otra índole como no disfrutar por la falta de tiempo en su día a día para dedicárselo al cuidado de los ejemplares.

Esto indica que es necesario realizar más prácticas de este tipo en la universidad, destacando su importancia al alumnado, para fomentar que sean realizadas en las aulas de educación 
infantil, motivando a los niños y niñas hacia el conocimiento de los insectos. La $6^{\mathrm{a}}$ afirmación, que posee una media superior a 4 indica que consideran que han aprendido sobre la biología de esta especie, ya que han tenido que observar su ciclo de vida tomando datos para posteriormente poder mostrar los resultados en su trabajo.

La $7^{\text {a }}$ afirmación estaba redactada de forma negativa y afirmaba que no es necesario mantener seres vivos en Educación Infantil, por lo que la media inferior a 2 puntos muestra que la mayoría no están de acuerdo con tal afirmación y consideran necesario tener seres vivos en Educación Infantil, lo que ya viene recomendado en el currículo de Educación Infantil (Orden $\mathrm{ECI} / 3960 / 2007)$.

Respecto a las dos últimas afirmaciones ( $8^{\mathrm{a}}$ y $9^{\mathrm{a}}$ afirmación), ambas con medias superiores a 4 puntos, indican que la mayoría tendrían gusanos de seda en las aulas de Educación Infantil en su futura labor docente y que aplicarían la investigación científica para estudiar la especie, fomentando la ciencia en las primeras etapas educativas, uno de los principales objetivos de este trabajo.

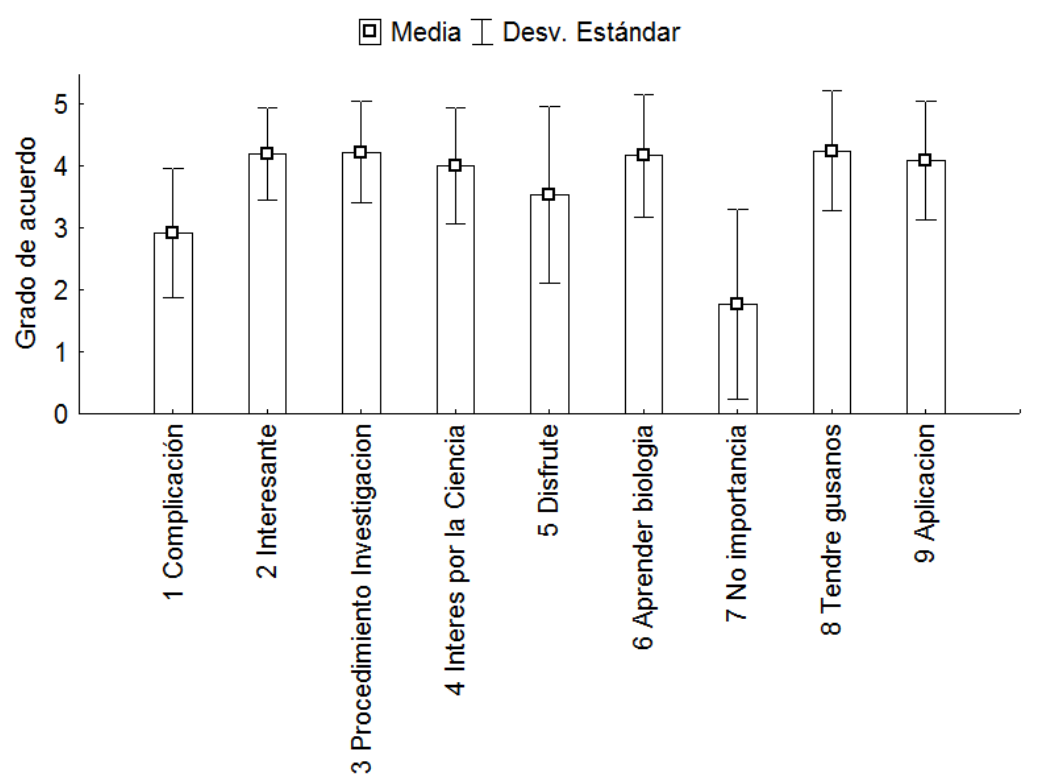

Figura 4. Resultados del cuestionario realizado al alumnado en el que podían expresar su grado de acuerdo con cada afirmación entre 1 y 5 ( $N=70$ estudiantes). Fuente: Propia.

El resultado global del cuestionario muestra que el desarrollo del proyecto ha sido positivo respecto a los fines perseguidos y que trabajar la metodología científica en el Grado de Educación Infantil puede hacerse de forma práctica para que posteriormente puedan aplicar este modelo en su futura profesión de maestros y maestras de Educación Infantil.

\section{Conclusiones}

El aprendizaje por investigación o por indagación está reconocido como una de las metodologías efectivas para el aprendizaje de las ciencias (Rocard, 2007; Caamaño, 2012; Brevins y Price, 2016; 
Romero-Ariza, 2017), pero para que estas metodologías lleguen a la escuela hay que comenzar por la universidad, en los grados de educación. Este proyecto muestra una metodología que ha servido para fomentar el acercamiento a la ciencia, mediante su práctica, en el alumnado del grado de Educación Infantil, con resultados positivos.

En primer lugar, se ha expuesto la metodología y los resultados científicos que se pueden obtener mediante el estudio de esta especie, que es fácil de mantener en cautividad, lo que puede servir de guía para plantear investigaciones tanto en la universidad, en diferentes grados (Grado de Educación Primaria, Grado de Biología), como en los institutos y escuelas (Primaria, Secundaria, Bachillerato), adaptando la complejidad a las distintas etapas educativas. Por lo tanto, los gusanos de seda constituyen un recurso ideal para realizar una investigación de aula, supliendo uno de los principales obstáculos del profesorado para aplicar la indagación en el aula, como es la falta de propuestas didácticas aplicables (Abril, Ariza, Quesada y García, 2014).

Por otra parte, se han mostrado los resultados de los trabajos realizados por el alumnado, en el que una elevada proporción diseña investigaciones, planteando interrogantes, tomando datos y obteniendo conclusiones, dando como resultado en algunos casos a sencillos artículos científicos, lo que implica la capacidad de desarrollar investigaciones. Aunque existe un porcentaje de trabajos que no muestra una metodología planificada, debido a que el aprendizaje por indagación es complejo para los estudiantes (Toma, Greca, y Meneses-Villagrá, 2017), influyendo el grado de asistencia del profesor (Alfieri et al., 2011) y especialmente en este caso de una indagación abierta sobre una temática concreta, donde los estudiantes tienen la libertad de plantear sus propios interrogantes e investigaciones. Se recomendaría en este caso, un seguimiento más estrecho del desarrollo del trabajo de cada grupo y analizar los diseños experimentales antes de su puesta en práctica (Holbrook y Kolodner, 2000). Por otra parte, lo positivo es que el estudio de esta especie permite plantear preguntas científicamente investigables, que suelen ser difíciles de plantear en otros escenarios (Ferrés-Gurt, 2017).

Respecto al diseño de actividades para trabajar la ciencia con los gusanos de seda en Educación Infantil, los resultados son positivos, ya que la mayoría ha planteado interrogantes y ha diseñado una metodología científica en la escuela. Sin embargo, como se ha mostrado en resultados, algunos trabajos no plantean interrogantes y exponen un seguimiento más sencillo de la especie, con actividades de observación, lo que muestra la dificultad que tienen los estudiantes, en algunos casos, para planificar actividades educativas basadas en la indagación, como se ha encontrado en otros estudios. Toma, Greca, y Meneses-Villagrá, (2017) solicitaron unidades didácticas basadas en la indagación a alumnado del grado de Maestro en Educación Primaria y casi el 67 \% del alumnado no diseñó unidades con elementos de la indagación.

Aunque el aprendizaje por investigación es complicado de desarrollar y planificar por el alumnado de distintas etapas educativas en general, el implicado en este proyecto considera que ha sido interesante, que ha asimilado el proceso que se sigue en una investigación aprendiendo sobre la biología de la especie estudiada, lo que ha contribuido a aumentar su interés por la ciencia. Además, consideran que es importante tener seres vivos en las aulas de Educación Infantil y que, en su futura etapa laboral como docentes de Educación Infantil, tendrían gusanos de seda en las 
aulas y fomentarían la aplicación del método científico en su alumnado.

Es necesario acercar la ciencia y la naturaleza a las aulas de Educación Infantil (García-Carmona, Criado y Cañal, 2014), fomentar la observación, que aprendan a plantear hipótesis, predicciones y tratar de resolverlas, aprovechando la curiosidad de los niños y niñas, siendo el aprendizaje por indagación uno de los más apropiados (Cutler et al., 2012; Harlen, 2013). Pero para conseguirlo hay que comenzar por acercar la ciencia y la naturaleza a los estudiantes universitarios (Gil Quilez et al., 2008; Torres-Porras et al., 2017). Los gusanos de seda se muestran como un recurso apropiado para trabajar la indagación y acercar los seres vivos a las aulas, tanto en la universidad como en la escuela, propiciando un estudio a lo largo de varios meses y no algo momentáneo o puntual. Los gusanos de seda ya captaron la atención de Maria-Sibylla Merian, naturalista alemana del siglo XVII que en su juventud se dedicó a estudiar esta especie (Merian, 1705) y a partir de esta inquietud continuó con el estudio de otras mariposas y sus ciclos, convirtiéndose en una entomóloga de referencia.

\section{Agradecimientos}

Agradecer la colaboración del alumnado y las sugerencias de los revisores que han permitido mejorar sustancialmente el artículo.

\section{Referencias bibliográficas}

Abril, A. M., Ariza, M. R., Quesada, A., y García, F. J. (2014). Creencias del profesorado en ejercicio y en formación sobre el aprendizaje por investigación. Revista Eureka sobre Enseñanza y Divulgación de las Ciencias, 11, 22-33.

Acevedo Díaz, J.A. (2004). Reflexiones sobre las finalidades de la enseñanza de las ciencias: educación científica para la ciudadanía. Revista Eureka sobre Enseñanza y Divulgación de las Ciencias, 1(1), 3-15.

Aragón Núñez, L., Jiménez Tenorio, N., Eugenio Gozalbo, M., y Vicente Martorell, J. J. (2016). Acercar la ciencia a la etapa de infantil: experiencias educativas en torno a talleres desde el Grado de Maestro en Educación Infantil. Revista Ibero-americana de Educación, 72, 105-128.

Bentley, M.L., y Auldridge, T. (2017). Worm Spit: Integrating Curriculum Through a Study of Silk and the Amazing Silk Worm. En M. P. Mueller, D. J. Tippins, A. J. Stewart (Eds.), Animals and Science Education: Ethics, Curriculum and Pedagogy (pp. 1-13). New York: Springer.

Bevins S., y Price, G. (2016). Reconceptualising inquiry in science education. International Journal of Science Education, 38(1), 17-29.

Briones Fernández, C., y Torres-Porras, J. (2016). Motivación del alumnado de Educación Preescolar en la enseñanza de las Ciencias. Revista Digital de Investigación Educativa Conect@2, 15, 58-81.

Caamaño, A. (2012). La investigación escolar es la actividad que mejor integra el aprendizaje de los diferentes procedimientos científicos. En E. Pedrinaci, A. Caamaño, P. Cañal, A. de Pro (Eds.), 11 ideas clave: El desarrollo de la competencia científica (pp. 127-146). Barcelona: Graó. 
Cantó Doménech, J., de Pro Bueno, A., y Solbes, J. (2016). ¿Qué ciencias se enseñan y cómo se hace en las aulas de educación infantil? La visión de los maestros en formación inicial. Enseñanza de las Ciencias, 34, 3, 25-50. http://dx.doi.org/10.5565/rev/ensciencias. 1870

Cañal, P. (1999). Investigación escolar y estrategias de enseñanza por investigación. Revista Investigación en la Escuela, (38), 15-36.

Cañal, P. (2006). La alfabetización científica en la infancia. Aula de Infantil, 33, 5-9.

Cortés Gracia, A.L., y de la Gándara Gómez, M. (2007). La construcción de problemas en el laboratorio durante la formación del profesorado: una experiencia didáctica. Enseñanza de las Ciencias, 25(3), 435-450.

COSCE (2011). Informe ENCIENDE: Enseñanza de las Ciencias en la didáctica escolar para edades tempranas en España. Madrid.

Cruz-Guzmán, M., García-Carmona, A., y Criado, A.M. (2017). Aprendiendo sobre los cambios de estado en educación infantil mediante secuencias de pregunta-predicción- comprobación experimental. Enseñanza de las Ciencias, 35, 3, 175-193. doi: https://doi.org/10.5565/ rev/ensciencias. 2336

Cutler, K., Bersani, C., Hutchins, P., Bowne, M., Lash, M., Kroeger, J., Brokmeier, S., Venhuizen, L., y Black, F. (2012). Laboratory Schools as Places of Inquiry: A Collaborative Journey for Two Laboratory Schools. Early Education and Development, 23(2), 242-258. doi: https://doi.org/ 10.1080/10409289.2012.647609

D’Angelo, E., y Medina, A. (1997). La asamblea en Educación Infantil: su relación con el aprendizaje y con la construcción de la autonomía. Revista Investigación en la Escuela, (33), 79-88.

Duschl, R.A., Schweingruber, H.A., y Shouse, A.W. (2006). Taking science to school: Learning and teaching science in grades K-8. Washington, DC: National Academies Press.

Eshach, H., y Fried, M.N. (2005). Should science be taught in early childhood? Journal of Science Education and Technology, 14(3), 315-336.

Fernández-Oliveras, A., Molina-Correa, V., y Oliveras, M.L. (2016). Estudio de una propuesta lúdica para la educación científica y matemática globalizada en infantil. Revista Eureka sobre Enseñanza y Divulgación de las Ciencias, 13(2), 373-383.

Ferrés-Gurt, C. (2017). El reto de plantear preguntas científicas investigables. Revista Eureka sobre Enseñanza y Divulgación de las Ciencias, 14(2), 410-426.

French, L. (2004). Science as the center of a coherent, integrated early childhood curriculum. Early Childhood Research Quarterly, 19, 138-149.

García-Barros, E. (1992). Fases preimaginales, distribución y ciclo vital de Coscinia romeii Sagarra, 1924 (Lepidoptera, Arctiidae). Eos, 68(2), 137-145.

García-Carmona, A., Criado, A.M., y Cañal, P. (2014). Alfabetización científica en la etapa 3-6 años: un análisis de la regulación estatal de enseñanzas mínimas. Enseñanza de las Ciencias, 32(2), 131-149. doi: https://doi.org/10.5565/rev/ensciencias.817

Gil, M.J., Martínez, M.B. y Cordero, S. (2017). Grabaciones de situaciones de aula para la formación del profesorado. Ápice. Revista de Educación Científica, 1(1), 58-73. doi: https://doi. org/10.17979/arec.2017.1.1.2005 
Gil Quilez, M.J., Martínez, M.B., de la Gándara, M., Calvo, J.M., y Cortés, A. (2008). De la universidad a la escuela: no es fácil la indagación científica. Revista Interuniversitaria de Formación del Profesorado, 63(22,3), 81-100.

Gomez-Montilla, C., y Ruiz-Gallardo, J.R. (2016). El rincón de la ciencia y la actitud hacia las ciencias en Educación Infantil. Revista Eureka sobre Enseñanza y Divulgación de las Ciencias, 13(3), 643-666.

Harlen, W. (2013). Inquiry-based learning in science and mathematics. Review of Science, Mathematics and ICT Education, 7(2), 9-33.

Holbrook, J., y Kolodner, J. L. (2000). Scaffolding the development of an inquiry-based (science) classroom. En B. J. Fishman y S. F. O'Connor-Divelbiss (Eds.), Proceedings of the Fourth International Conference of the Learning Sciences (pp. 221-227). Ann Arbor: University of Michigan.

Lederman, J. S., Lederman, N. G., Bartos, S. A., Bartels, S. L., Antink, A., y Schwartz, R. S. (2014). Meaningful assessment of learners' understanding about scientific inquiry- The views about scientific inquiry (VASI) questionnaire. Journal of Research in Science Teaching, 51(1), 65-83.

Martens, M.L. (1999). Productive questions: tools for supporting constructivist learning. Science and Children, 36, (8), 24-27.

Martin-Hansen, L. (2002). Defining inquiry. The science teacher, 69(2), 34.

Martínez-Ramírez, A. (2002). La vida del gusano de seda. Jaén: Asociación ecologista guardabosques de Jódar.

Merian, M. S. (1705). Metamorphosis insectorum surinamensium. Amsterdam.

Minner, D, Levy, A., y Century, J. (2010). Inquiry-based science instruction-What is it and does it matter? Results from a research synthesis years 1984 to 2002. Journal of Research in Science Teaching, 47(4), 474-496.

Nayfeld, I., Brenneman, K., y Gelman, R. (2011). Science in the classroom: Finding a balance between autonomous exploration and teacher-led instruction in preschool settings. Early Education and Development, 22(6), 970-988. doi: https://doi.org/10.1080/10409289.2010.507496

Osborne J., y Dillon J. (2008). Science education in Europe: Critical reflections. (Vol. 13). London: The Nuffield Foundation.

Pedrinaci, E., Caamaño, A., Cañal, P., y de Pro, A. (2013). El desarrollo de la competencia científica. 11 ideas clave. Barcelona: Graó.

Rocard, M. (2007). Science education now: A renewed pedagogy for the future of Europe. Bruselas, Comisión Europea.

Rodríguez, O. A., Vargas, M. J., Ventura, M. A., Martínez, M. A., Rodríguez, M. J., Ehsan, M., y Lara, V. F. M. (2012). Manual de Sericultura en Hidalgo, principios Básicos. Primera edición. Universidad Politécnica de Francisco I. Madero: México.

Romero-Ariza, M. (2017). El aprendizaje por indagación, ¿existen suficientes evidencias sobre sus beneficios en la enseñanza de las ciencias? Revista Eureka sobre Enseñanza y Divulgación de las Ciencias, 14(2), 286-299.

Sanmartí, N., y Márquez, C. (2012). Enseñar a plantear preguntas investigables. Alambique. Didáctica de las Ciencias Experimentales, 70, 27-36. 
Siry, C. (2013). Exploring the complexities of children's inquiries in science: Knowledge production through participatory practices. Research in Science Education, 4, 2407-2430.

Spektor-Levy, O., Kesner, Y., y Mevarec, Z. (2013). Science and scientific curiosity in preschool. The teacher's point of view. International Journal of Science Education, 35(13), 2226-2253.

Tamir, P., y García, M. (1992). Características de los ejercicios de prácticas de laboratorio incluidos en los libros de texto de ciencias utilizados en Cataluña. Enseñanza de las Ciencias, 10(1), 3-12.

Toma, R.B., Greca, I.M., y Meneses-Villagrá, J.A. (2017). Dificultades de maestros en formación inicial para diseñar unidades didácticas usando la metodología de indagación. Revista Eureka sobre Enseñanza y Divulgación de las Ciencias, 14(2), 442-457.

Torres-Porras, J., Alcántara, J., Arrebola, J.C., Rubio, S.J., y Mora, M. (2017). Trabajando el acercamiento a la naturaleza de los niños y niñas en el Grado de Educación Infantil. Crucial en la sociedad actual. Revista Eureka sobre Enseñanza y Divulgación de las Ciencias, 14(1): 258-270.

Torres-Porras, J. (2017). Acercamiento a la naturaleza y a los seres vivos. En R. Mérida Serrano, J. Torres-Porras y J. Alcántara Manzanares (Eds.), Didáctica de las ciencias experimentales en educación infantil (pp. 131-157). Madrid: Editorial Síntesis.

Torres-Porras, J., y Villamandos de la Torre, F. (2015). Aplicación del método científico en el laboratorio. Investigando el proceso de crecimiento en organismos pluricelulares. Educación, Arte, Comunicación: Revista Académica Investigativa y Cultural, 5, 12-17.

Windschitl, M. (2003). Inquiry projects in science teacher education: What can investigative experiences reveal about teacher thinking and eventual classroom practice? Science Education, 87(1), 112-143. 